\title{
Opioid Modulation of Value-Based Decision-Making in Healthy Humans
}

\author{
Marie Eikemo*,1,2,3, Guido Biele ${ }^{1,4}$, Frode Willoch ${ }^{5}$, Lotte Thomsen' and Siri Leknes ${ }^{1,6}$ \\ 'The Department of Psychology, University of Oslo, Oslo, Nonway; ${ }^{2}$ Norwegian Center for Addiction Research, Department of Clinical Medicine, \\ University of Oslo, Oslo, Nonway; ${ }^{3}$ Division of Mental Health and Addiction, Oslo University Hospital, Oslo, Nonway; ${ }^{4}$ Department of Child Health, \\ Norwegian Institute of Public Health, Oslo, Norway; ${ }^{5}$ The Department of Basic Medical Sciences, University of Oslo, Oslo, Norway; ${ }^{6}$ The \\ Intervention Centre, Oslo University Hospital, Oslo, Norway
}

\begin{abstract}
Modifying behavior to maximize reward is integral to adaptive decision-making. In rodents, the $\mu$-opioid receptor (MOR) system encodes motivation and preference for high-value rewards. Yet it remains unclear whether and how human MORs contribute to value-based decision-making. We reasoned that if the human MOR system modulates value-based choice, this would be reflected by opposite effects of agonist and antagonist drugs. In a double-blind pharmacological cross-over study, 30 healthy men received morphine (I 0 mg), placebo, and the opioid antagonist naltrexone (50 mg). They completed a two-alternative decision-making task known to induce a considerable bias towards the most frequently rewarded response option. To quantify MOR involvement in this bias, we fitted accuracy and reaction time data with the drift-diffusion model (DDM) of decision-making. The DDM analysis revealed the expected bidirectional drug effects for two decision subprocesses. MOR stimulation with morphine increased the preference for the stimulus with high-reward probability (shift in starting point). Compared to placebo, morphine also increased, and naltrexone reduced, the efficiency of evidence accumulation. Since neither drug affected motor-coordination, speed-accuracy trade-off, or subjective state (indeed participants were still blinded after the third session), we interpret the MOR effects on evidence accumulation efficiency as a consequence of changes in effort exerted in the task. Together, these findings support a role for the human MOR system in value-based choice by tuning decision-making towards high-value rewards across stimulus domains.
\end{abstract}

Neuropsychopharmacology (2017) 42, 1833-1840; doi:I0.1038/npp.2017.58; published online 12 April 2017

\section{INTRODUCTION}

Adaptive decision-making involves successfully predicting appetitive outcomes, as well as selectively approaching rewards (Fields and Margolis, 2015). So far, research on neurochemical modulators of value-based decision-making in humans has centered around contributions of the dopamine, noradrenaline and serotonin systems (eg, Rogers, 2011). Nonetheless, maladaptive decision-making is commonly observed in clinical populations with disrupted $\mu$ opioid receptor (MOR) function, eg, substance dependence (Lubman et al, 2009) and chronic pain (Apkarian et al, 2004). Further, evidence from rodent models demonstrate $\mu$ opioid modulation of important subprocesses of value-based decision-making (Laurent et al, 2015). In rats, MOR agonism primarily enhances preference for high-value rewards, as indicated by measures of consumption (DiFeliceantonio et al, 2012) as well as by effort exerted to achieve rewards (eg, progressive ratio schedules (Zhang et al, 2003)). Blockade of

\footnotetext{
*Correspondence: M Eikemo, Norwegian Centre for Addiction Research, University of Oslo, PO BOX 1039 Blindern, Oslo 0315, Norway, Tel: +47 233689 76, Fax: +47 233689 86,

E-mail:m.h.eikemo@medisin.uio.no

Received 14 November 2016; revised 20 February 2017; accepted 7 March 2017; accepted article preview online 15 March 2017
}

MORs shows the opposite effects (Cleary et al, 1996). Overall, evidence from pharmacological manipulation studies indicate that the rodent MOR system regulates preference and valuation in the food, sexual (Mahler and Berridge, 2012) and social (Moles et al, 2004) domains.

In humans, the MOR system is best known as a moderator of pain and pleasure (eg, Leknes and Tracey, 2008). Molecular imaging has demonstrated endogenous MOR activity during pain relief and certain types of reward (eg, Boecker et al, 2008; Colasanti et al, 2012; Henriksen and Willoch, 2008; Hsu et al, 2013). Hence, MOR signaling in humans likely affects value-based decision-making by altering the value of rewards and punishments. For instance, substance abusers' preference for drug cues above all other rewards may be mediated by MOR system dysfunction (Ghitza et al, 2010). Preliminary psychopharmacological evidence from our lab showed that in healthy men, MOR agonism increased, and antagonism decreased, reward motivation and preference, specifically for the high-value stimuli, ie, the sweetest drink (Eikemo et al, 2016) and the most attractive opposite sex faces (Chelnokova et al, 2014; Chelnokova et al, 2016). These findings align well with previous evidence that MOR antagonism reduces preference for high-value food rewards (high-calorie foods; Ziauddeen et al, 2013). 
Here, we investigated how the MOR system modulates value-based choice in healthy humans. In a psychopharmacological study with a three-way repeated-measures design, 30 opioid-naive men received the MOR agonist morphine (10 mg per-oral), the non-selective opioid antagonist naltrexone $(50 \mathrm{mg})$, and placebo on 3 separate days. Since only the MOR is strongly affected by both drugs, we assume that behaviors influenced bidirectionally reflect drug effects on MOR and not other opioid receptors. Participants completed a two-alternative signal detection task with asymmetric rewards (Figure 1). The task is designed to induce a considerable response bias towards the most frequently rewarded response option (Pizzagalli et al, 2005). The response bias is markedly reduced in patients with disruptions of reward processing caused by, eg, depression (Pizzagalli et al, 2008b).

To assess MOR system involvement in the processes underpinning value-based choice, we fitted trial-by-trial accuracy and reaction time data with a Bayesian implementation of the drift-diffusion model (DDM) of decisionmaking. The DDM is a well-established computational model used to study cognitive components of twoalternative decisions (Ratcliff and McKoon, 2008). It has provided valuable information about how decision subprocesses are influenced by drugs (Pedersen et al, in press; Van Ravenzwaaij et al, 2012), psychopathology (Banca et al, 2015), and by task properties such as time restriction or reward schedule (Mulder et al, 2012; Wiech et al, 2014).

The DDM describes the decision processes as a gradual accumulation of the difference in evidence for the two choice options (Ratcliff and McKoon, 2008). The decision begins at a starting point $(z)$ in-between two decision boundaries, where the boundary separation parameter $(a)$ quantifies the speed-accuracy trade-off; Figure 2. The efficiency of evidence accumulation is captured by the drift rate $(v)$ parameter. A response is initiated once sufficient evidence has been accumulated to reach one of the two decision boundaries.

We expected the starting point parameter to reflect the response bias induced by the skewed reward schedule of the present task (Mulder et al, 2012). Further, since MOR drugs modulate both preference and motivation in rodents, we hypothesized that MOR manipulations in healthy humans would modulate the shift in starting point and/or the efficiency of the evidence accumulation. We reasoned that behaviors modulated by the endogenous MOR system in the healthy human brain would show bidirectional drug modulation, as indicated by the linear contrast (morphine $>$ placebo $>$ naltrexone).

\section{MATERIALS AND METHODS}

\section{Study Design and Participants}

The study was conducted in a double-blind, placebo controlled manner. Tasks and drug conditions were pseudo-randomized and counterbalanced. Participants were 32 healthy men with normal or corrected-to-normal vision. One participant tested positive on the opiate urine screening (MOP Opiate300 Test Strip; SureScreen Diagnostics Ltd, Derby, UK) and another failed to complete all sessions, yielding a final group of $n=30$ (mean age 26.9, range 20-36). Exclusion criteria included: history of or current psychiatric/

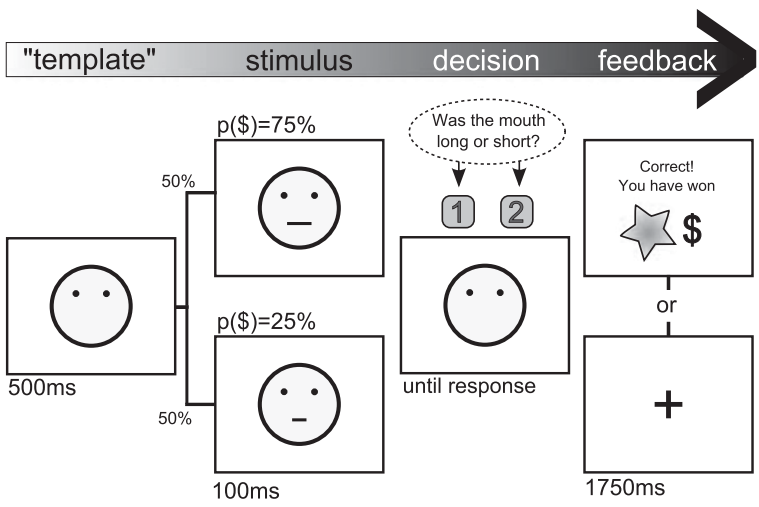

Figure I Experimental task. Participants were presented with schematic faces and instructed to identify which of two alternative mouths was shown (eg, short or long mouth). We developed three equally ambiguous stimulus sets for use in the three sessions to avoid any learning effects (Supplementary Figure SI). Unknown to the participant, correct identification of one of the stimulus alternatives led to a monetary reward (NOKI, $\sim 15$ cents) three times more often than the other stimulus (75 vs 25\% reward probability). The most frequently rewarded response option is considered the high-value stimulus. Non-rewarded and incorrect trials were followed by a fixation cross. Altogether 300 trials were divided into three blocks. The differences between the face stimuli have been inflated for illustrative purposes

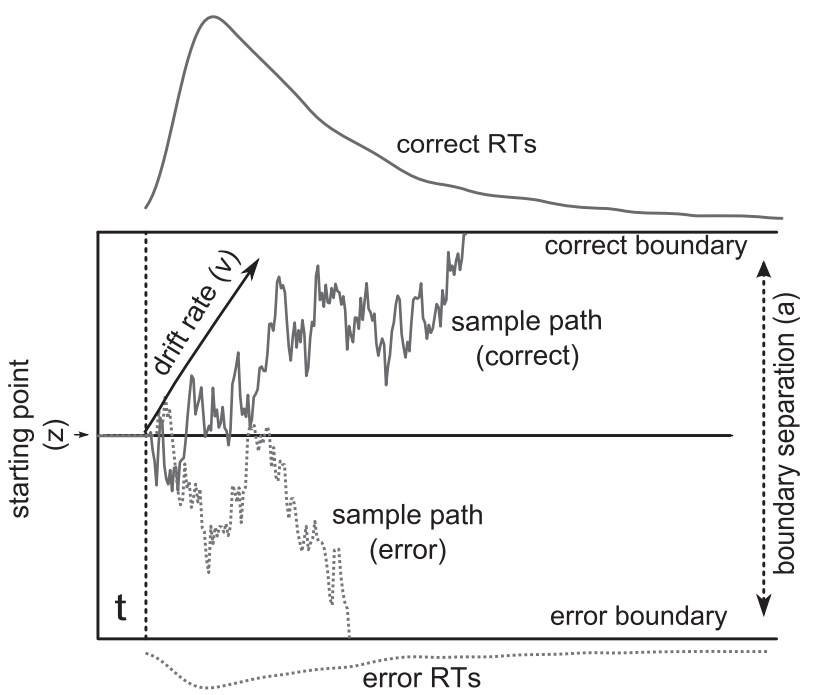

Figure 2 The drift-diffusion model of decision-making. The model assumes that relative evidence for two decision alternatives is accumulated until a decision boundary is reached. The figure illustrates key parameters of the model. The drift rate ( $v$ ) represents how much evidence is on average accumulated per time unit, and can indicate task difficulty, ability or effort exerted in the task. The jagged lines represent sample paths (the upward sloping line for a correct response; the downward sloping path for an erroneous response). The starting point (z), also called bias, provides information about whether the decision maker is favoring one option before trial onset. The figure here shows no bias. The boundary separation (a) indicates the speed-accuracy trade-off: the larger the separation, the more decision makers prefer accuracy over speed. The parameter $t$ captures nondecision time needed for stimulus processing and response execution. The figure also shows typical response time distributions (error and correct trials) for a larger number of choices.

medical illness, prior drug dependence/addiction, use of medication except antihistamines and contraceptives, complex allergies. Alcohol and drug use was assessed during screening (Supplementary Information). 


\section{Time Line}

Participants were tested on three different days with a minimum inter-session interval of seven days. Each session lasted approximately $3 \mathrm{~h}$ during which participants completed a battery of experimental tasks. An optimal test interval (60-120 min after drug intake) was deduced by comparing the time of maximal bioavailability and half-life of oral morphine and naltrexone. The monetary reward task was conducted on average $\sim 90$ min post drug administration. Participants were reimbursed 400-500 NOK (\$60-70) per session, depending on task performance. The experimental procedures were approved by the Regional Ethics Committee (2011/1337/REK Sør-ØstD). Participants were informed that they could withdraw from the study at any time. Participants were asked to sustain from alcohol since the evening before each test session, to sustain from eating an hour before testing, and not to drive a vehicle for $6 \mathrm{~h}$ after drug administration. Participants were not allowed to consume caffeine or nicotine during the test session.

\section{Drug Administration}

Morphine is an opioid agonist with high affinity to the $\mu$-receptor. To minimize subjective drug effects, we chose 10 mg per-oral morphine (Morfin, Nycomed Pharma, Asker, Norway). Previous reports have shown only weak subjective effects to larger doses (eg, Zacny and Lichtor, 2008). Naltrexone is a non-selective opioid antagonist with high affinity to $\mu$ - and $\kappa$-opioid receptors. We used $50 \mathrm{mg}$ per-oral naltrexone (Adepend, Orpha-Devel, Purkersdorf, Austria), a standard dosage that blocks more than $90 \%$ of MORs (Weerts et al, 2013). Both drugs induce a plasma concentration plateau $\sim 1 \mathrm{~h}$ after intake (eg, Lugo and Kern, 2002; Verebey et al, 1976). Placebo pills were cherry-flavored breath mints that were visually matched to morphine and naltrexone pills. A small amount of the flavored placebo pills were added to the drug dosages, to avoid recognition of medication taste. Participants were asked to swallow, rather than chew, the pills.

\section{Control Measurements}

Subjective state (including mood: happiness, anxiousness, irritability, and feeling good) ratings were collected (i) before drug ingestion (baseline); (ii) $60 \mathrm{~min}$ after drug ingestion; (iii) $~ 40$ min into testing; and (iv) after completion of all tasks using electronic visual analog scales implemented in MatLab R2012a (Mathworks, Natic, USA). A motorcoordination task was performed $\sim 100 \mathrm{~min}$ after drug administration (Supplementary Information).

\section{Value-Based Decision-Making Task}

Reward behavior was tested with a two-alternative forcedchoice task (adapted from Pizzagalli et al, 2005, Figure 1). In each of 300 trials, a schematic face with no mouth is presented for $500 \mathrm{~ms}$, followed by a brief presentation of one of two mouth stimuli $(100 \mathrm{~ms})$. The two stimuli are slightly different in one dimension, eg, length. The task is to correctly identify the mouth stimulus displayed in each trial. The two mouth stimuli are equiprobable and presented in a random order within each block. Participants are informed that correct responses can sometimes, but not every time, lead to a monetary reward. The reward message is presented immediately after a rewarded trial and replaced by a fixation cross after $1750 \mathrm{~ms}$. Incorrect and unrewarded trials are followed by a fixation cross. Unknown to the participant, the task is based on a skewed reinforcement schedule. One of the two stimuli is associated with a larger probability of reward (75\% when the correct answer is provided), than the other stimulus (25\% reward probability following a correct trial). The most frequently rewarded response option is considered a high-value option. Participants performed a different version of the task in each session to avoid learning effects (see Supplementary Information, Supplementary Figure S1).

Behavioral data from this task has by large been assessed within a signal detection framework using block-wise accuracy (aggregated in three 100-trial blocks). While the bias (criterion) in a traditional signal detection framework is calculated as the ratio of hits for each response option, the DDM integrates both accuracy and reaction time data in the estimation of the decision parameters. The behavioral biases induced by the task can be reflected in the drift rate $(v)$ and starting point $(z)$, parameters reflecting the efficiency of evidence accumulation and a priori preference for one response option respectively. The key advantage of a DDM analysis of the data is that it allows for a clear discrimination of information processing efficiency and speed-accuracy trade-off, whereas the discriminability parameter in the signal detection framework reflects both these parameters. The tasks were presented on a 20" PC monitor using E-prime software (version 2.0; Psychology Software Tools, Inc., Pittsburgh, Pennsylvania, USA).

\section{Control Data Analysis}

To test effects of drug manipulations on motor function and mood we used Bayesian hierarchical implementations of generalized linear models in Stan via RStan (Stan Development Team, 2014; see Supplementary Information for details).

\section{Drift-Diffusion Model Analysis}

Reaction time and accuracy data was fit with the DDM of decision-making. We used the HDDM toolbox (Wiecki et al, 2013), which allows for hierarchical modeling of DDM parameters in a Bayesian framework. To capture the withinsubjects design of our experiment, we used a regression approach to model effects of the drug manipulation and of learning over blocks in each session. Because the aim of the current study was to examine the bidirectional effects of drugs relative to the placebo condition, we directly incorporated the relevant contrasts into the regression model (three drug conditions times three block conditions, see Supplementary Information for details).

The DDM included the drift rate $(v)$, starting point $(z)$, boundary separation $(a)$, and non-decision time $(t)$ parameters. The drift rate parameter is often interpreted as an index of task difficulty. However, in a within-subjects design with constant stimulus quality as employed here, drift rate can be interpreted as the efficiency of the signal processing, reflecting attention allocated or effort exerted during the 
task. Because we had no hypotheses concerning the nondecision time parameter of the DDM, $t$, this parameter was kept constant across conditions. Additional DDM parameters describing trial-by-trial variation in non-decision time, drift rate, and bias were not tested because parameter recovery experiments showed that these are difficult to estimate reliably on the individual level, and because their estimation is computationally expensive (Wiecki et al, 2013).

To estimate contrasts of model parameters in a hierarchical manner, we estimated a group-level mean and SD of model parameters, which served as priors for individual level parameters. The effects reported are group-level means. Contrasts for drug effects on DDM parameters are presented as posterior density plots that represent posterior beliefs about parameter values (Figure 5), commonly used for reporting results from Bayesian analyses. To visualize if and how well the model and the estimated parameters captured the data, we performed a posterior predictive check (Gelman et al, 2014), using the fitted model parameters to simulate data and then comparing these to the observed data in order to look for systematic discrepancies (Supplementary Information). The final DDM analysis included data from 27 participants (for details about data exclusion and Bayesian estimation see Supplementary Information).

\section{RESULTS}

\section{Control Measures}

As expected since drug doses were optimized to avoid subjective drug effects, Bayesian regression analyses revealed no credible effects on mood (Figure 3; see Supplementary Information and Supplementary Table S3 for details). Importantly, successful blinding (34\% correct guesses at debriefing in the third session) confirmed that drug administration of morphine and naltrexone was not associated with sedation, subjective high or other effects likely to affect task performance. Further, comparable performance across drug conditions on a motor-coordination control task indicated that neither of the drugs impaired ability to perform the task (Supplementary Table S4).

\section{Descriptive Statistics}

Inspection of means and variance information for accuracy, and reaction time data for each drug condition indicated the presence of the expected behavioral bias towards the most frequently rewarded stimulus. Overall, participants were faster and more accurate on high-reward stimulus trials (Figure 4).

\section{Drift-Diffusion Model Results}

Baseline task performance. To evaluate how the DDM parameters would reflect the behavioral effects of the task, we first examined performance in the placebo condition (blockwise results are presented in Supplementary Information, Supplementary Figure S2, and Supplementary Table S1). A shift in the starting point of the evidence accumulation $(z)$ was evident in the first block and increased over time. This result replicates the key finding of previous signal detection analysis of this task, namely that participants increasingly learn to prefer the most frequently rewarded response option (Pizzagalli et al, 2005), using computational modeling methodology. Estimation of the other relevant parameters revealed that both the drift rate $(v)$ and boundary separation (a) decreased over time, ie, from the first 100 trials (block 1) until the completion of the 300 trials. This likely reflects task fatigue. Note that comparable fatigue effects were observed in the two drug conditions as well.

Drug effects on decision parameters. The DDM revealed bidirectional drug effects on the rate of evidence accumulation, that is the drift rate $(v)$ : Opioid antagonism with
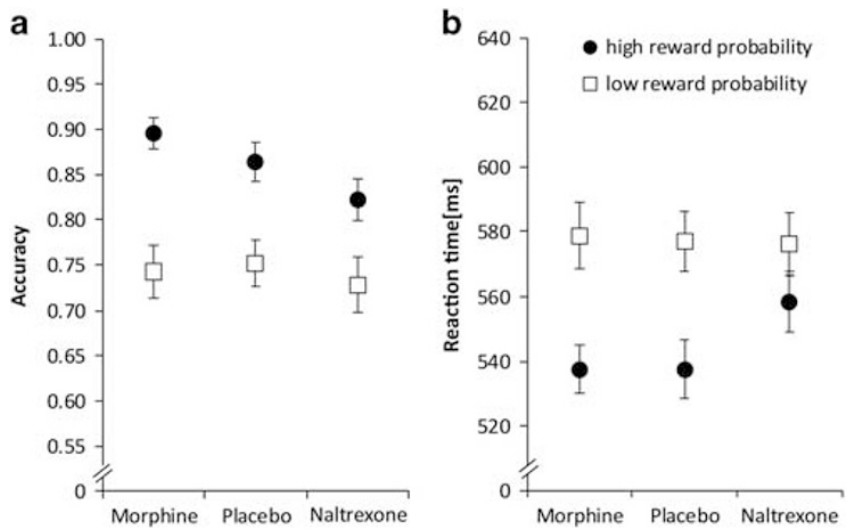

Figure 4 Descriptive data. Average accuracy (a) and reaction time (b) data presented by drug condition and stimulus type (high- and low-reward probability) for the 27 participants included in the final DDM analysis. Error bars represent within-subject SEM.
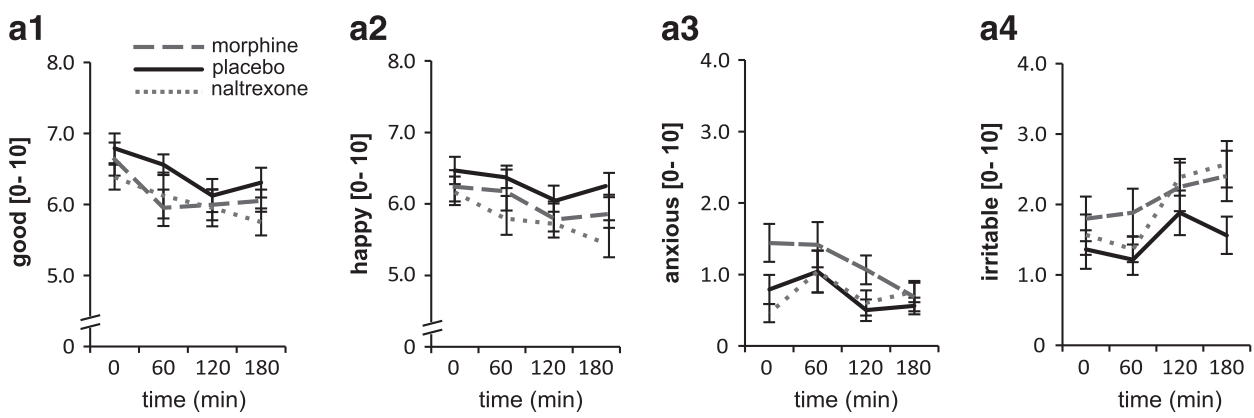

Figure 3 No credible drug effects were found for any of the mood types. For illustration, average ratings with within-subjects SEM are presented for all measurements throughout the test phase ( $x$-axis values refer to minutes after drug ingestion). 
naltrexone decreased the drift rate compared to the placebo condition (mean difference $\Delta(90 \% \mathrm{HDI})=0.17 \quad(-0.29$, -0.058), mean Cohen's $d=-0.46)$. The @@posterior probability of a lower drift rate in the naltrexone condition was $0.99(\mathrm{p}(\mathrm{N}<\mathrm{P}))$. In contrast, drift rate was increased following MOR agonism with morphine $(\Delta=0.126(-0.015$, $0.268), \mathrm{p}(\mathrm{M}>\mathrm{P})=0.93, d=0.30)$. The comparison of posterior distributions for the $\mathrm{M}>\mathrm{P}>\mathrm{N}$ contrast shows that the drift rate following MOR agonism was higher than after MOR antagonism with a posterior probability of 0.99 (Figure 5).

A similar drug effect pattern was found for the starting point parameter $(z)$. Across all drug conditions, the starting point was shifted towards the high-value option. Morphine increased the shift of the starting point towards the highreward boundary compared to the placebo control condition $(\Delta=0.041 \quad(-0.004,0.084), \mathrm{p}(\mathrm{M}>\mathrm{P})=0.94)$. While the starting point only tended to be less skewed towards the high-reward option in the naltrexone condition compared to placebo $(\Delta=0.032(-0.092,0.03,) ; \mathrm{p}(\mathrm{N}<\mathrm{P})=0.81)$, it was clearly decreased with naltrexone compared to morphine $(\Delta=0.073(-0.008,0.147), \mathrm{p}(\mathrm{M}>\mathrm{N})=0.94$.

a1
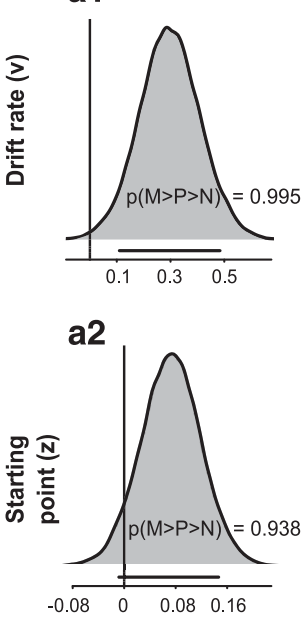

a3

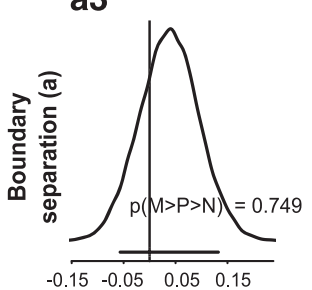

b1

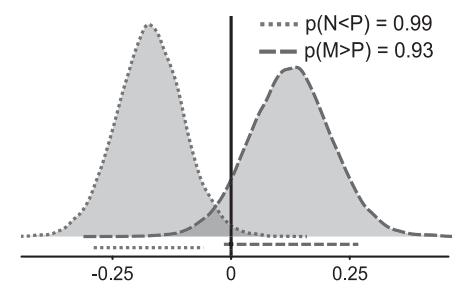

b2

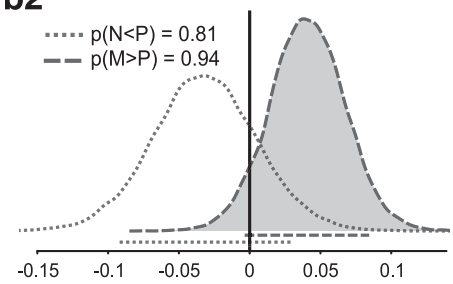

b3

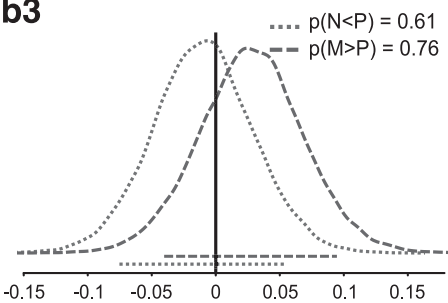

We found no credible support that the MOR system influences boundary separation (Figure 5), as evidenced by comparable parameter estimates for naltrexone and placebo $(\Delta=0.01 \quad(-0.075,0.54), \mathrm{p}(\mathrm{N}<\mathrm{P})=0.61, d=0.09) ;)$ and morphine and placebo $(\Delta=0.038 \quad(-0.040,0.95)$, $\mathrm{p}(\mathrm{M}>\mathrm{P})=0.76, d=0.34)$.

In sum, the computational modeling results support MOR system modulation of value-based decision-making by changes in both the starting point towards a stimulus associated with high-reward probability and the rate of the evidence accumulation (Figure $5 \mathrm{~d}$ and Supplementary Table S1).

\section{DISCUSSION}

Results from bidirectional drug manipulation of MORs provide evidence for MOR system modulation of value-based choice in healthy humans. As hypothesized, MOR blockade reduced the propensity to modulate behavior as a function of reward probability, primarily through lowered evidence accumulation rate compared to placebo. Conversely, MOR

c1

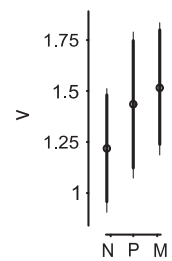

c2
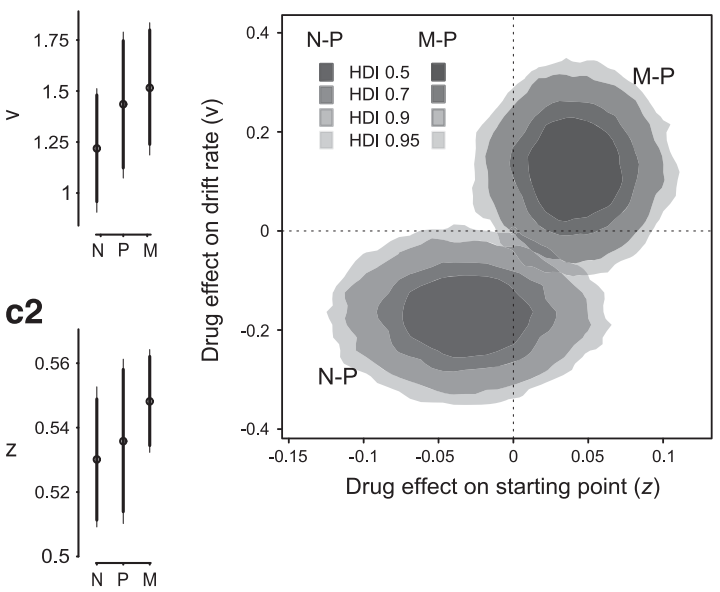

c3

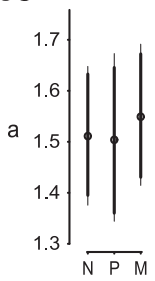


system activation with a non-sedative dose of morphine led to higher drift rate. Morphine also shifted the starting point of each decision closer towards the high-value boundary. Indeed, the agonist and antagonist drugs appear to 'pull' these two decision subprocesses in opposite directions compared to placebo.

When people develop a preference towards one response option, this is usually reflected in a shifted starting point of the decision process. The bidirectional MOR modulation of starting point observed here indicates human MOR system involvement in preference for high-value (high-probability) rewards. This finding is in line with rodent studies demonstrating that MOR stimulation increases while MOR blockade decreases preference specifically for high-value rewards such as sucrose, fat, and sex cues (Mahler and Berridge, 2012). Task-induced changes in starting point have previously been associated with brain activity in regions of a fronto-parietal network, including several MOR-rich cingulate and prefrontal areas (Henriksen and Willoch, 2008; Mulder et al, 2012).

MOR modulation of preference for the high-value response option was also manifest in changes of evidence accumulation efficiency (drift rate). Compared to placebo, morphine increased and naltrexone decreased accumulation efficiency in the task. Since stimulus quality was kept constant across counterbalanced tasks in the three sessions, we interpret the changes in drift rate as a reflection of the motivation to obtain reward. Higher motivation to earn money should increase attention and effort exerted in the task, thereby enhancing the efficiency of evidence accumulation. In principle, changes in drift rate could also result from drug effects on visual acuity, ie, by enhancing or disrupting overall stimulus perception. However, we find no evidence of drug effects on vision. MOR manipulations primarily affected accuracy for the high-reward option. Further, eyehand coordination performance was comparable across drug conditions. Viewing the drift rate changes as a reflection of altered attention and effort, is supported by rodent findings that MOR manipulation modifies the value an animal places on a reinforcer (DiFeliceantonio et al, 2012). Indeed, infusion of a MOR agonist into the nucleus accumbens increased rats' willingness to expend effort to obtain a reward, as measured by progressive ratio schedule (Zhang et al, 2003). Furthermore, injecting $\mu$-opioid drugs directly into mesolimbic structures such as the nucleus accumbens increases rats' incentive motivation (Berridge et al, 2009).

Notably, the present MOR drug effects on value-based choice occurred without credible changes in mood, subjective 'high', alertness or motor coordination. Debriefing revealed that participants performed at chance level when asked to identify the drugs at the end of the three sessions. Hence, drug effects on value-based choice are likely to reflect MOR modulation of decision subprocesses and not indirect effects due to subjective drug effects. Since both the starting point and bias measures were modulated bidirectionally, we assume that the effects are driven by the only known receptor strongly affected by both drugs (MOR and not KOR).

The computational modeling approach used here yields mechanistic information above and beyond what can be gleaned from descriptive data and signal detection analysis. Behavioral biases can have systematic effects on the response time distributions for errors and correct responses. These are captured by the DDM, but would be missed in analyses limited to accuracy data. The estimation of DDM parameters enabled assessment of drug effects on latent psychological processes that make up the decision process. The assumptions of the DDM, as a sequential sampling model of decision-making, have not only been shown to accurately describe behavioral results (Ratcliff and McKoon, 2008). Studies linking this model to neurophysiological (Gold and Shadlen, 2007; Shadlen and Newsome, 2001) and neuroimaging mechanisms of decision-making (Basten et al, 2010) indicate that the model can describe neurobiological mechanisms underpinning binary decisions.

In the healthy human brain, endogenous opioids are released to regulate pain (Henriksen and Willoch, 2008) and rejection (Hsu et al, 2013). Preliminary molecular imaging evidence also indicates opioid release during certain rewards: social acceptance (Hsu et al, 2013), exercise 'high' (Boecker et al, 2008) and after positive mood induction (Koepp et al, 2009). We have previously reported evidence that bidirectional MOR manipulations affected explicit liking and wanting of high-value rewards in the taste (Eikemo et al, 2016) and face perception (Chelnokova et al, 2014; Chelnokova et al, 2016) domains. Here, we corroborate and extend upon these findings by showing MOR modulation of implicit motivation and preference. Indeed, debriefing confirmed that the task's skewed reward schedule was unknown to participants even after three test sessions. Further, we extend the evidence of MOR reward modulation to a secondary reinforcer (money). These results support a general role of MORs across reward domains. Indeed, brain regions richly innervated by MORs such as ventral striatum, amygdala and perigenual anterior cingulate cortex have been consistently implicated in human neuroimaging studies of monetary and other rewards (McClure et al, 2004).

The current results in healthy participants also align well with studies showing impaired value-based decision-making across substance-use disorders (Lubman et al, 2009; Paulus, 2007) and with the growing evidence for dysregulation of MOR function across addictions (Ghitza et al, 2010; Mick et al, 2015). Indeed, addiction is characterized by a maladaptive preference for certain activities, such as drug abuse or pathological gambling, at the cost of all other rewards. Such exaggerated preference for drug cues predicted relapse to heroin (Lubman et al, 2009). Thus the increase in preference for high-value reward observed here after acute morphine administration may represent an innocuous precursor to the maladaptive drug preference commonly observed after chronic MOR stimulation with opioids, stimulants or alcohol (Colasanti et al, 2012; Mitchell et al, 2012). Notably, sustained MOR antagonist treatment has led to improvements in craving and other pathological behaviors across addictions (Lobmaier et al, 2011).

The meso-cortico-limbic dopamine (DA) and limbic MOR systems are co-located in the nucleus accumbens, ventral pallidum, and amygdala and are thought to play complementary and central roles in reward processing (Berridge et al, 2009). Thus, the present effects on effort exerted in the reward task may in part reflect MOR modulation of mesolimbic DA signaling (Johnson and North, 1992). The reward task used here also involves implicit learning of reward probabilities which likely recruits dopaminergic neurotransmission (Pessiglione et al, 2006). Indeed, inducing 
an antagonistic effect on DA was previously shown to reduce learning and preference for high-value reward in this task (Pizzagalli et al, 2008a). However, evidence from substantial rodent opioid research points to important, independent contributions of the MOR system to different aspects of reward processing, notably to reward valuation and 'liking' (eg, Berridge et al, 2009; Hnasko et al, 2005; Laurent et al, 2015). Accordingly, we speculate that MOR system modulation of value is the main mechanism underpinning the observed drug effects on starting point and drift rate.

Some limitations of the present study warrant consideration. Only male participants were tested, as opioid drugs have been shown to interact with female cyclic hormones (Smith et al, 1998). Since the present findings mirror results from rodent studies, we consider it likely that future studies will demonstrate similar MOR modulation of value-based decision-making in women. Although we do not explicitly control for intake of nicotine or caffeine in the present analysis, the within-subjects design renders systematic influences of these substances unlikely. Although it is a not limitation as such, we wish to stress that the present results indicate a reduction and not an elimination of reward responsiveness after naltrexone treatment. At $50 \mathrm{mg}$ per-oral, this opioid antagonist induces a high degree of MOR blockade in the healthy brain (>90\% of endogenous receptors; Weerts et al, 2013). Value-based choice is likely influenced by the MOR system in interaction with, eg, noradrenaline, serotonin, dopamine, and endocannabinoids (Rogers, 2011). Understanding how these systems together contribute to aversive and appetitive processes in healthy humans and psychopathology remains an important task for the future.

In sum, these results suggest that the human MOR system guides value-based choice by tuning decision-making towards high-value rewards. Further, the findings support a role for the human MOR system promoting adaptive strategies such as effort expenditure and preference for highly valuable rewards across stimulus domains. Knowledge of the basic affective mechanisms of the MOR system in healthy humans is a necessary foundation for development of improved, targeted treatments of the millions of patients whose MOR systems have been disrupted by chronic pain or substance dependence.

\section{FUNDING AND DISCLOSURE}

The study was supported by grants from the Norwegian Research Council (ES455867) and the South-Eastern Norway Regional Health Authority (2013053). The study is indirectly supported by Aleris Healthcare, Norway, financing the research position of Frode Willoch at the University of Oslo. The authors declare no conflict of interest.

\section{ACKNOWLEDGMENTS}

We thank Guro Løseth, Olga Chelnokova, Hedda Gjertsen, Jeppe Riegels, Ingunn Olsen for help with data collection. We thank Elisabeth Øiestad, Vigdis Vindenes and Liliana Bachs for help with determining medication doses and analyses of biological data. We thank Paul Whalen and Desirée Spronk for valuable comments on earlier versions of this manuscript.

\section{REFERENCES}

Apkarian AV, Sosa Y, Krauss BR, Thomas PS, Fredrickson BE, Levy RE et al (2004). Chronic pain patients are impaired on an emotional decision-making task. Pain 108: 129-136.

Banca P, Vestergaard MD, Rankov V, Baek K, Mitchell S, Lapa T et al (2015). Evidence accumulation in obsessive-compulsive disorder: the role of uncertainty and monetary reward on perceptual decision-making thresholds. Neuropsychopharmacology 40: 1192-1202.

Basten U, Biele G, Heekeren HR, Fiebach CJ (2010). How the brain integrates costs and benefits during decision making. Proc Natl Acad Sci 107: 21767-21772.

Berridge KC, Robinson TE, Aldridge JW (2009). Dissecting components of reward: 'liking', 'wanting', and learning. Curr Opin Pharmacol 9: 65-73.

Boecker H, Sprenger T, Spilker ME, Henriksen G, Koppenhoefer M, Wagner KJ et al (2008). The runner's high: opioidergic mechanisms in the human brain. Cereb Cortex 18: 2523-2531.

Chelnokova O, Laeng B, Eikemo M, Riegels J, Løseth G, Maurud H et al (2014). Rewards of beauty: the opioid system mediates social motivation in humans. Mol Psychiatry 19: 746-747.

Chelnokova O, Laeng B, Løseth G, Eikemo M, Willoch F, Leknes S (2016). The $\mu$-opioid system promotes visual attention to faces and eyes. Soc Cogn Affect Neurosci 11: 1902-1909.

Cleary J, Weldon DT, O'Hare E, Billington C, Levine AS (1996). Naloxone effects on sucrose-motivated behavior. Psychopharmacology 126: 110-114.

Colasanti A, Searle GE, Long CJ, Hill SP, Reiley RR, Quelch D et al (2012). Endogenous opioid release in the human brain reward system induced by acute amphetamine administration. Biol Psychiatry 72: 371-377.

DiFeliceantonio AG, Mabrouk OS, Kennedy Robert T, Berridge KC (2012). Enkephalin surges in dorsal neostriatum as a signal to eat. Curr Biol 22: 1918-1924.

Eikemo M, Løseth GE, Johnstone T, Gjerstad J, Willoch F, Leknes S (2016). Sweet taste pleasantness is modulated by morphine and naltrexone. Psychopharmacology 233: 3711-3723.

Fields HL, Margolis EB (2015). Understanding opioid reward. Trends Neurosci 38: 217-225.

Gelman A, Carlin JB, Stern HS, Dunson DB, Vehtari A, Rubin DB. Bayesian Data Analysis. 3rd edn. CRC Press: Boca Raton, 2014.

Ghitza UE, Preston KL, Epstein DH, Kuwabara H, Endres CJ, Bencherif B et al (2010). Brain mu-opioid receptor binding predicts treatment outcome in cocaine-abusing outpatients. Biol Psychiatry 68: 697-703.

Gold JI, Shadlen MN (2007). The neural basis of decision making. Annu Rev Neurosci 30: 535-574.

Henriksen G, Willoch F (2008). Imaging of opioid receptors in the central nervous system. Brain 131: 1171-1196.

Hnasko TS, Sotak BN, Palmiter RD (2005). Morphine reward in dopamine-deficient mice. Nature 438: 854-857.

Hsu DT, Sanford BJ, Meyers KK, Love TM, Hazlett KE, Wang H et al (2013). Response of the $\mu$-opioid system to social rejection and acceptance. Mol Psychiatry 18: 1211-1217.

Johnson SW, North RA (1992). Opioids excite dopamine neurons by hyperpolarization of local interneurons. J Neurosci 12: 483-488.

Koepp MJ, Hammers A, Lawrence AD, Asselin MC, Grasby PM, Bench CJ (2009). Evidence for endogenous opioid release in the amygdala during positive emotion. Neuroimage 44: 252-256.

Laurent V, Morse AK, Balleine BW (2015). The role of opioid processes in reward and decision-making. Br J Pharmacol 172: 449-459.

Leknes S, Tracey I (2008). A common neurobiology for pain and pleasure. Nat Rev Neurosci 9: 314-320.

Lobmaier PP, Kunoe N, Gossop M, Waal H (2011). Naltrexone depot formulations for opioid and alcohol dependence: a systematic review. CNS Neurosci Ther 17: 629-636. 
Lubman DI, Yücel M, Kettle JWL, Scaffidi A, MacKenzie T, Simmons JG et al (2009). Responsiveness to drug cues and natural rewards in opiate addiction: Associations with later heroin use. Arch Gen Psychiatry 66: 205-213.

Lugo RA, Kern SE (2002). Clinical pharmacokinetics of morphine. J Pain Palliative Care Pharmacother 16: 5-18.

Mahler SV, Berridge KC (2012). What and when to 'want'? Amygdala-based focusing of incentive salience upon sugar and sex. Psychopharmacology 221: 407-426.

McClure SM, York MK, Montague PR (2004). The neural substrates of reward processing in humans: the modern role of fMRI. Neuroscientist 10: 260-268.

Mick I, Myers J, Ramos AC, Stokes PRA, Erritzoe D, Colasanti A et al (2015). Blunted endogenous opioid release following an oral amphetamine challenge in pathological gamblers. Neuropsychopharmacology 41: 1742-1750.

Mitchell JM, O’Neil JP, Janabi M, Marks SM, Jagust WJ, Fields HL (2012). Alcohol consumption induces endogenous opioid release in the human orbitofrontal cortex and nucleus accumbens. Sci Transl Med 4: 116ra6.

Moles A, Kieffer BL, D'Amato FR (2004). Deficit in attachment behavior in mice lacking the mu-opioid receptor gene. Science 304: 1983-1986.

Mulder MJ, Wagenmakers E-J, Ratcliff R, Boekel W, Forstmann BU (2012). Bias in the brain: a diffusion model analysis of prior probability and potential payoff. J Neurosci 32: 2335-2343.

Paulus MP (2007). Decision-making dysfunctions in psychiatryaltered homeostatic processing? Science 318: 602-606.

Pedersen ML, Frank MJ, Biele G (2016). The drift diffusion model as the choice rule in a reinforcement learning model. Psychon Bull Rev (epub ahead of print).

Pessiglione M, Seymour B, Flandin G, Dolan RJ, Frith CD (2006). Dopamine-dependent prediction errors underpin reward-seeking behaviour in humans. Nature 442: 1042-1045.

Pizzagalli DA, Evins AE, Schetter EC, Frank MJ, Pajtas PE, Santesso DL et al (2008a). Single dose of a dopamine agonist impairs reinforcement learning in humans: behavioral evidence from a laboratory-based measure of reward responsiveness. Psychopharmacology 196: 221-232.

Pizzagalli DA, Iosifescu D, Hallett LA, Ratner KG, Fava M (2008b). Reduced hedonic capacity in major depressive disorder: evidence from a probabilistic reward task. J Psychiatr Res 43: 76-87.

Pizzagalli DA, Jahn AL, O'Shea JP (2005). Toward an objective characterization of an anhedonic phenotype: a signal-detection approach. Biol Psychiatry 57: 319-327.
Ratcliff R, McKoon G (2008). The diffusion decision model: theory and data for two-choice decision tasks. Neural Comput 20: 873-922.

Rogers RD (2011). The roles of dopamine and serotonin in decision making: evidence from pharmacological experiments in humans. Neuropsychopharmacology 36: 114-132.

Shadlen MN, Newsome WT (2001). Neural basis of a perceptual decision in the parietal cortex (area LIP) of the rhesus monkey. J Neurophysiol 86: 1916.

Smith YR, Zubieta J-K, Carmen MGd, Dannals RF, Ravert HT, Zacur HA et al (1998). Brain opioid receptor measurements by positron emission tomography in normal cycling women: relationship to luteinizing hormone pulsatility and gonadal steroid hormones. J Clin Endocrinol Metab 83: 4498-4505.

Stan Development Team (2014). RStan: the R interface to Stan, Version 2.5. Available at http://mc-stan.org/interfaces/rstan.html.

Van Ravenzwaaij D, Dutilh G, Wagenmakers EJ (2012). A diffusion model decomposition of the effects of alcohol on perceptual decision making. Psychopharmacology 219: 1017-1025.

Verebey K, Volavka J, Mule SJ, Resnick RB (1976). Naltrexone: disposition, metabolism and effects after acute and chronic dosing. Clin Pharmacol Ther 20: 315-328.

Weerts EM, McCaul ME, Kuwabara H, Yang X, Xu X, Dannals RF et al (2013). Influence of OPRM1 Asn40Asp variant (A118G) on [11C]carfentanil binding potential: preliminary findings in human subjects. Int J Neuropsychopharmacol 16: 47-53.

Wiech K, Vandekerckhove J, Zaman J, Tuerlinckx F, Vlaeyen JW, Tracey I (2014). Influence of prior information on pain involves biased perceptual decision-making. Curr Biol 24: R679-R681.

Wiecki TV, Sofer I, Frank MJ (2013). HDDM: hierarchical bayesian estimation of the drift-diffusion model in python. Front Neuroinform 7: 14 .

Zacny JP, Lichtor SA (2008). Within-subject comparison of the psychopharmacological profiles of oral oxycodone and oral morphine in non-drug-abusing volunteers. Psychopharmacology 196: $105-116$.

Zhang M, Balmadrid C, Kelley AE (2003). Nucleus accumbens opioid, GABAergic, and dopaminergic modulation of palatable food motivation: contrasting effects revealed by a progressive ratio study in the rat. Behav Neurosci 117: 202-211.

Ziauddeen H, Chamberlain SR, Nathan PJ, Koch A, Maltby K, Bush $\mathrm{M}$ et al (2013). Effects of the mu-opioid receptor antagonist GSK1521498 on hedonic and consummatory eating behaviour: a proof of mechanism study in binge-eating obese subjects. Mol Psychiatry 18: 1287-1293.

Supplementary Information accompanies the paper on the Neuropsychopharmacology website (http://www.nature.com/npp) 